\title{
Um Mapeamento Sistemático sobre as Iniciativas para Promover o Pensamento Computacional
}

\author{
Júlia S. B. Ortiz ${ }^{1}$, Roberto Pereira ${ }^{1}$ \\ ${ }^{1}$ Programa de Pós-Graduação em Informática \\ Universidade Federal do Paraná - UFPR. Curitiba, Brasil. \\ jubathke@gmail.com, rpereira.infegmail.com
}

\begin{abstract}
Resumo. Nos últimos anos muitas iniciativas foram realizadas para ensinar Pensamento Computacional, porém são poucos trabalhos que visam identificar as ações realizadas nestas pesquisas. Este artigo apresenta um mapeamento sistemático da literatura que cobriu as pesquisas que tiveram como objetivo promover o desenvolvimento do Pensamento Computacional, publicadas no período 2007-2017, em Inglês ou Português, em uma base nacional e três bases internacionais, com o propósito de identificar e analisar as ações efetivamente realizadas. Dentre os artigos selecionados identificamos: os públicos de alunos alcançados pelas iniciativas, os países que mais publicaram, os temas abordados como conteúdos das iniciativas, as ferramentas utilizadas como apoio, entre outros. Como resultado da análise, tendências e oportunidades para pesquisas futuras foram identificadas.
\end{abstract}

\begin{abstract}
In recent years many initiatives have been carried out to teach Computational Thinking, but there are few studies that identify and analyze the actions taken in these researches. This paper presents a systematic mapping of the literature covering the research published in the period 2007-2017, in English or Portuguese, in one Brazilian and three international basis, in order to identify and analyze research actions intended to promote the development of Computational Thinking. From the selected papers, we identified the audience of students that were reached by the initiatives, countries that have published in this area, the topics addressed as a theme of the initiatives and the tools they used. As a result, trends and opportunities for future research have been identified.
\end{abstract}

\section{Introdução}

Pensamento computacional (PC) foi descrito por [Wing 2006] como a habilidade de utilizar conceitos fundamentais da computação (como abstração) na resolução de problemas relacionados ou não com a computação. Embora este termo tenha sido citado por outros pesquisadores há bastante tempo, como [Papert 1980], foi após o artigo de Wing que o termo ganhou visibilidade e passou a atrair a atenção de pesquisadores de diversas áreas, inclusive de fora da Computação. No artigo, a autora comenta situações cotidianas nas quais usamos o PC sem perceber, e defende que essa é uma habilidade fundamental para todos, não somente para cientistas da computação.

As instituições Computer Science Teachers Association (CSTA) e International Society for Technology in Education (ISTE) em 2010 definiram $^{1}$ PC como um processo

\footnotetext{
${ }^{1}$ Disponível em: http://www.iste.org/docs/ct-documents/computational-thinking-operational-definitionflyer.pdf
} 
VII Congresso Brasileiro de Informática na Educação (CBIE 2018)

Anais do XXIX Simpósio Brasileiro de Informática na Educação (SBIE 2018)

de solução de problemas que inclui (mas não está limitado) às seguintes características: formular problemas de maneira que permita utilizar o computador e outras ferramentas para ajudar a resolvê-los, organizar e analisar os dados de maneira lógica, representar dados por meio de abstrações como modelos e simulações, automatizar soluções por meio do pensamento algorítmico, identificar, analisar e implementar possíveis soluções com o objetivo de alcançar a melhor combinação de eficiência e eficácia na utilização de recursos, e generalizar e transferir este processo de resolução para uma ampla variedade de problemas. Além destas características, foram também listadas como habilidades compreendidas pelo PC: coleta de dados, análise de dados, representação de dados, decomposição de problemas, abstração, algorítmos e procedimentos, automação, paralelização e simulação [Barr and Stephenson 2011].

Nos últimos anos o PC se tornou foco de muitas pesquisas ao redor do mundo, com iniciativas para desenvolver essas habilidades com alunos de diferentes níveis de formação, abordando diferentes conteúdos, com diferentes estratégias e ferramentas. Assim, este artigo apresenta um mapeamento sistemático da literatura com o objetivo de fornecer um panorama dos trabalhos publicados que apresentem iniciativas para promover $o$ PC enquanto uma habilidade de raciocínio. O mapeamento cobriu trabalhos publicados no período 2007-2017, buscando verificar se as iniciativas para promover o PC estão se tornando frequentes, quais países estão pesquisando este tema, quais níveis de formação escolar estão sendo alcançados por essas iniciativas, entre outros. Por meio do mapeamento foi detectado que diversas pesquisas já foram realizadas com este objetivo, que a quantidade de pesquisas têm aumentado após 2015, que o país com maior quantidade de publicações é Estados Unidos e que, embora exista grande quantidade e frequência de publicações, PC enquanto tema de pesquisa ainda é novo, apresentando muitas oportunidades e desafios para futuros trabalhos. Nas próximas seções serão apresentados os trabalhos relacionados, o método aplicado para o mapeamento, com seus resultados e discussões.

\section{Trabalhos Relacionados}

Para verificar se a realização deste mapeamento apontaria informações desconhecidas sobre o estado da arte deste tema de pesquisa, foram analisados mapeamentos e revisões sistemáticas da literatura que investigaram questões relacionadas ao PC realizados nos últimos dois anos e publicados nos eventos Simpósio Brasileiro de Informática na Educação (SBIE) e Congresso Brasileiro de Informática na Educação (CBIE).

Zanetti et al. (2016) realizaram um mapeamento sistemático para investigar o que estava sendo feito no Brasil sobre PC no contexto de ensino de programação. Esse mapeamento cobriu artigos dos eventos Simpósio Brasileiro de Informática na Educação (SBIE), Workshop de Informática na Escola (WIE), Workshop sobre Educação em Informática (WEI) e Workshop de Ensino em Pensamento Computacional, Algoritmos e Programação (WAlgProg), no período entre 2007 e 2015. Liz et al. (2016) realizaram um mapeamento sistemático sobre como o PC estava sendo estimulado e avaliado no Brasil, buscando artigos dos eventos SBIE, WIE, WEI, WAlgProg e na Revista de Novas Tecnologias na Educação (RENOTE) publicados até julho de 2016.

Carvalho et al. (2017) realizaram uma revisão sistemática sobre os objetos de aprendizagem utilizados para realizar as atividades embasadas pelo PC, cobrindo artigos 
VII Congresso Brasileiro de Informática na Educação (CBIE 2018)

Anais do XXIX Simpósio Brasileiro de Informática na Educação (SBIE 2018)

dos eventos SBIE, WIE, WEI, da Revista Brasileira de Informática na Educação (RBIE) e da RENOTE, no período entre 2012 e 2017. Avila et al. (2017) realizaram uma revisão sistemática sobre metodologias de avaliação de habilidades ou dimensões do PC. Os autores buscaram artigos publicados nas bases digitais online IEEE, ACM, EBSCOhost e Science Direct, de 2011 até agosto de 2016. Bordini et al. (2017), por sua vez, realizaram uma revisão sistemática sobre iniciativas de PC no ensino fundamental e médio, buscando artigos nas bases digitais online IEEE, ACM, Scopus, Science Direct e Springer, publicados entre 2012 e agosto de 2016.

O mapeamento sistemático descrito neste artigo se difere dos acima mencionados pois pesquisou artigos realizados dentro e fora do Brasil, em bibliotecas digitais online internacionais e nacionais, publicados em uma janela de tempo maior (de 2007 até novembro de 2017), buscando todas as pesquisas que relatam atividades realizadas para promover o PC, independente do público alvo e do tipo de atividade desenvolvida.

\section{Método}

O objetivo do mapeamento sistemático da literatura foi selecionar os artigos que efetivamente apresentam e discutem práticas realizadas para promover o desenvolvimento do PC como uma habilidade de raciocínio. Este processo foi conduzido seguindo as diretrizes de [Petersen et al. 2015] e compreendeu as fases de busca de artigos e aplicação do primeiro, segundo e terceiro filtros para, então, realizar a extração dos dados e conduzir as análises.

As perguntas de pesquisa definidas para este mapeamento foram: P1: As pesquisas em PC estão se tornando frequentes? Algum país se destaca na quantidade de pesquisas?; P2: Quais os níveis de formação educacional abordados por essas iniciativas?; P3: As iniciativas abordam alunos em contexto desafiador?; P4: Quais conteúdos foram utilizados como tema para as atividades conduzidas?; P5: Quais ferramentas foram utilizadas nas atividades?; P6: Qual a duração das iniciativas realizadas junto ao público?.

Para a busca, foram considerados os artigos que possuíssem os termos "Pensamento Computacional" ou "Computational Thinking” em seus títulos, disponíveis nas bases digitais online $\mathrm{ACM}^{2}$, IEEE Explore ${ }^{3}$, Springer ${ }^{4}$ e Comissão Especial em Informática na Educação $(\mathrm{CEIE})^{5}$, publicados entre 2007 e 2017. A escolha das bibliotecas considerou a relevância das mesmas para a pesquisa em Ciência da Computação. A busca retornou 468 artigos, sendo 230 na ACM, 102 na IEEE, 75 na Springer e 61 na CEIE.

No primeiro filtro, com base no título e no resumo dos artigos, foram incluídos os que relatavam a realização de projetos (oficinas, cursos, entre outros) com o objetivo de promover o PC em qualquer contexto. Artigos que não apresentassem ou discutissem os resultados de alguma ação foram excluídos. Após o primeiro filtro, restaram 222 artigos, sendo 118 da ACM, 44 da IEEE, 31 da Springer e 29 da CEIE.

No segundo filtro, realizado com a leitura completa dos artigos, foram aplicados os critérios de exclusão: C1: Pesquisas que relatam revisão sistemática ou outros tipos que não caracterizam intervenções práticas com os alunos; C2: Pesquisas duplicadas (quando

\footnotetext{
${ }^{2}$ Disponível em: https://dl.acm.org/dl.cfm

${ }^{3}$ Disponível em: https://ieeexplore.ieee.org

${ }^{4}$ Disponível em: https://www.springer.com/

${ }^{5}$ Disponível em: http://www.br-ie.org/pub/index.php/index
} 
VII Congresso Brasileiro de Informática na Educação (CBIE 2018)

Anais do XXIX Simpósio Brasileiro de Informática na Educação (SBIE 2018)

havia dois ou mais artigos sobre uma mesma iniciativa, foram mantidos os artigos mais recentes ou completos); C3: Pesquisas que não estavam em Inglês ou Português; C4: Pesquisas não disponíveis para acesso integral; C5: Pesquisas que apresentam apenas propostas para integrar o PC em uma grade curricular; C6: Pesquisas que propuseram métodos para avaliar o PC; C7: Pesquisas que somente avaliam frameworks, metodologias e ferramentas para desenvolver o PC; C8: Pesquisas que propõem ferramentas para o desenvolvimento do PC, sem práticas com o objetivo de ensinar os alunos e C9: Pesquisas que relatam instruir professores a aplicar atividades relacionadas ao PC. Foram selecionados 62 artigos, sendo 33 da ACM, 10 da IEEE, 2 da Springer e 17 da CEIE.

No terceiro filtro ocorreu a aplicação de critérios de qualidade, visando manter apenas artigos que permitissem a análise e resposta das perguntas de pesquisa. Como o objetivo do mapeamento era identificar pesquisas que apresentem práticas realizadas para promover o desenvolvimento do PC, foram desconsiderados os artigos que não caracterizavam as práticas apresentadas, impossibilitando o entendimento, a extração dos dados e a replicação das mesmas em outros contextos. Permaneceram na amostra final 46 artigos, sendo 23 da ACM, 8 da IEEE, 2 da Springer e 13 da CEIE. Para a etapa de extração de dados foi desenvolvido um template contendo as perguntas de extração e respostas préformatadas para cada uma delas, com o objetivo de padronizar o processo e responder as perguntas de pesquisa.

\section{Resultados}

Foi disponibilizado no apêndice A as tabelas que contêm a lista dos artigos integrantes da amostra final do mapeamento, contendo ID e referência completa. Para facilitar a identificação, nas respostas às perguntas de pesquisa cada artigo foi referenciado por seu código ID. Nos momentos em que a quantidade de artigos a ser referenciada excede 8 , os mesmos foram listados nas notas de rodapé.

P1: As pesquisas em PC estão se tornando frequentes? Algum país se destaca na quantidade de pesquisas? Embora o mapeamento tenha buscado artigos publicados a partir de 2007, o artigo mais antigo selecionado é de 2009, realizado por pesquisadores dos Estados Unidos, indicando que pesquisas para promover o PC são relativamente novas, não tendo ainda completado uma década. É somente a partir de 2015 que aparecem artigos publicados por outros países. Durante todo o período observado apenas 5 pesquisas foram realizadas por pesquisadores de diferentes países em conjunto [18, 26, 27, 28 e 38] e, nestes casos, os artigos foram contabilizados para todos os países participantes. A Tabela 1 apresenta a quantidade total de artigos por ano e por país. Analisando especificamente os últimos três anos, é possível observar que no primeiro deles há um aumento significativo na quantidade total de pesquisas enquanto nos dois últimos a quantidade se mantém constante. Analisando a quantidade de pesquisas realizadas por país, neste mapeamento os Estados Unidos possui maior representatividade, seguido pelo Brasil devido a inclusão de pesquisas em Português.

P2: Quais são os níveis de formação educacional abordados por essas iniciativas? Para responder esta pergunta, as informações foram normalizadas e categorizadas de acordo com a idade e nível escolar. Dos 46 artigos, 20 trabalharam com alunos do

\footnotetext{
6*: diz respeito aos países Canadá, Chile, Escócia, Índia, Israel e Taiwan, todos com uma pesquisa cada.
} 
VII Congresso Brasileiro de Informática na Educação (CBIE 2018)

Anais do XXIX Simpósio Brasileiro de Informática na Educação (SBIE 2018)

Tabela 1. Artigos publicados por ano e país.

\begin{tabular}{|c|c|c|c|c|c|c|c|c|c|c|}
\hline & $\mathbf{2 0 0 9}$ & $\mathbf{2 0 1 0}$ & $\mathbf{2 0 1 1}$ & $\mathbf{2 0 1 2}$ & $\mathbf{2 0 1 3}$ & $\mathbf{2 0 1 4}$ & $\mathbf{2 0 1 5}$ & $\mathbf{2 0 1 6}$ & $\mathbf{2 0 1 7}$ & Total \\
\hline EUA & 2 & 1 & 4 & 2 & 3 & 2 & 3 & 2 & 2 & $\mathbf{2 1}$ \\
\hline Brasil & - & - & - & - & - & - & 4 & 6 & 5 & $\mathbf{1 5}$ \\
\hline Itália & - & - & - & - & - & - & 1 & - & 3 & $\mathbf{4}$ \\
\hline Espanha & - & - & - & - & - & - & - & 1 & 2 & $\mathbf{3}$ \\
\hline Alemanha & - & - & - & - & - & - & 1 & - & 1 & $\mathbf{2}$ \\
\hline Outros* & - & - & - & - & - & - & - & 6 & - & $\mathbf{6}$ \\
\hline Argentina & - & - & - & - & - & - & - & - & 1 & $\mathbf{1}$ \\
\hline Total & $\mathbf{2}$ & $\mathbf{1}$ & $\mathbf{4}$ & $\mathbf{2}$ & $\mathbf{3}$ & $\mathbf{2}$ & $\mathbf{9}$ & $\mathbf{1 5}$ & $\mathbf{1 4}$ & \\
\hline
\end{tabular}

ensino fundamental ${ }^{6}$, com idade entre 6 e 15 anos, 8 trabalharam com estudantes do ensino médio ${ }^{7}$, com idade entre 15 e 18 anos e 4 pesquisas trabalharam com alunos do ensino fundamental e ensino médio juntos [11, 27, 40 e 45]. Outros estudos abordaram alunos fora da educação básica: 8 artigos trabalharam com estudantes de graduação ou pós-graduação em cursos de diversas áreas ${ }^{8}, 4$ artigos trabalharam com estudantes de graduação em cursos relacionadas à Ciência da Computação [6, 7, 8 e 11], e 1 artigo trabalhou com alunos da Educação de Jovens e Adultos (EJA) [30]. Analisando os públicos explorados nos países de maior representatividade observamos que os EUA abordaram a maior diversidade de públicos, apresentando pesquisas em todas as categorias exceto na EJA $^{9}$, e o Brasil realizou todas iniciativas na educação básica, exceto 1 conduzida na EJA.

P3: As iniciativas abordam alunos em contexto desafiador? Somente 8 artigos mencionam ter envolvido estudantes em contextos desafiadores, dos quais 3 foram realizados nos EUA [5, 10 e 34], 1 na Alemanha [18], 1 no Chile [28], 1 no Brasil [30], 1 no Nepal $^{10}$ [35] e 1 na Itália [43]. Dessas iniciativas, 2 artigos mencionam estudantes com limitações cognitivas: um abordou estudantes com Transtorno do Espectro do Autismo [28] e outro abordou estudantes com síndrome de Asperger, paralisia cerebral ou dislexia [10]. Os outros 6 artigos mencionam limitações relacionadas à condição financeira e social da família [5, 18, 30, 35 e 43], alunos de regiões com dificuldades de acesso à escola [18 e 35] e alunos ou escolas situadas em área rural [34 e 35].

P4: Quais conteúdos foram utilizados como tema para as atividades conduzidas? Muitas pesquisas apresentam mais de um conteúdo para tematizar as atividades destinadas à promover o PC. Os assuntos relacionados à Ciência da Computação como bancos de dados, programação, algoritmos, entre outros, foram os mais mencionados aparecendo em $21 \operatorname{artigos}^{11}$. Em seguida, em 4 artigos aparecem atividades focando no desenvolvimento de jogos [33, 40, 42 e 43], e em outros 4 aparecem atividades multidisciplinares [11, 16, 45 e 46]. A Matemática foi mencionada em 3 artigos [13, 20 e 34]. Assuntos não relacionados diretamente com a computação também foram mencionados: Bioinformática [1 e 2], Ciência [34 e 35], Física [1 e 14], Química [1 e 14], Música [3 e 14], Engenharia [7 e 34], Arquitetura [40], Artes [18], Biologia [27], Business [8], Geometria [9], Jornalismo [5], Gerontologia [4] e Literatura Inglesa [17]. Analisando os temas mais trabalhados nos

\footnotetext{
${ }^{6}$ Ensino Fundamental: 9, 12, 16, 18, 22, 23, 24, 27, 28, 31, 32, 34, 35, 37, 38, 41, 42, 43, 45 e 46.

${ }^{7}$ Ensino Médio: 14, 15, 17, 19, 20, 21, 29 e 33.

${ }^{8}$ Graduação ou pós em diversas áreas: 1, 2, 3, 4, 5, 13, 25, 36 e 40.

${ }^{9}$ No Canadá: Adults Learning Center, e nos EUA: General Education Development.

${ }^{10}$ Uma das pesquisas conduzidas pelos EUA foi realizada no Nepal.

${ }^{11}$ Assuntos de Ciência da Computação: 6, 10, 12, 15, 19, 21, 22, 23, 24, 25, 26, 28, 29, 30, 31, 33, 36, $37,38,43$ e 44 .
} 
VII Congresso Brasileiro de Informática na Educação (CBIE 2018)

Anais do XXIX Simpósio Brasileiro de Informática na Educação (SBIE 2018)

países de maior representatividade, observamos que os Estados Unidos foi o país com atividades em maior quantidade e diversidade de temas, e que essa diversidade é percebida desde os primeiros trabalhos mapeados (2009). Os demais países focaram em tematizar suas atividades somente em assuntos relacionados à Ciência da Computação, atividades multidisciplinares, desenvolvimento de jogos e matemática.

P5: Quais ferramentas foram utilizadas nas atividades? Muitos artigos mencionam ter utilizado mais de uma ferramenta na realização das atividades, e diversas ferramentas foram mencionadas. As mais citadas foram: Computação Desplugada ${ }^{12}$ (do Inglês Computer Science Unppluged) mencionada em 16 artigos, a ferramenta Scratch ${ }^{13}$, mencionada em 12 artigos $^{14}$ e Kit de robótica ${ }^{15}$ mencionado em 8 artigos. Considerando os artigos dos EUA e Brasil, Computação Desplugada foi a ferramenta mais utilizada, mencionada em $60 \%$ das pesquisas brasileiras, e em $24 \%$ das americanas, que se destacam por adotar também maior variedade de ferramentas nas pesquisas conduzidas.

P6: Qual foi a duração das iniciativas realizadas junto ao público? 3 artigos não mencionam esta informação. Nos demais, os dados referentes a essa pergunta foram extraídos conforme mencionado pelos autores (alguns optaram por informar a quantidade de encontros, outros por semana, etc.) e agrupados em categorias. Os artigos mencionam ter duração variando desde 1 dia até 1 ano. Os períodos 1 semestre $^{16}$ e $4-7$ encontros $^{17}$ foram os mais reportados, com 12 e 11 artigos, respectivamente. Os demais foram: 1-3 encontros [9, 18 e 39], 8-15 encontros [23 e 28], 2-4 semanas [5, 33 e 35], 5-7 semanas [31, 36 e 38], 8-15 semanas [11, 22, 25, 34, 45 e 46] e 1 ano [16]. Analisando as pesquisas por país, observamos que 65\% dos artigos dos EUA duraram de 8-15 semanas ou mais, enquanto $46 \%$ das pesquisas brasileiras foram realizadas entre 1-7 encontros.

\section{Discussão}

Por meio dos dados apresentados é possível observar que a pesquisa em PC ainda está em estágio inicial, ainda não tendo completado uma década mesmo no cenário internacional quando se trata de resultados de iniciativas práticas (a primeira pesquisa, dos EUA, foi publicada em 2009). No Brasil, a publicação de artigos com resultados de práticas explicitamente situadas pelos autores no tema de PC é ainda mais nova, datando de 2015. Porém, embora recente, o Brasil já realizou quantidade relevante de pesquisas na área, neste mapeamento atrás somente dos Estados Unidos. Entretanto, embora o Brasil tenha apresentado boa representatividade, devido a inclusão do idioma Português no mapeamento, ao comparar as configurações das iniciativas propostas pelo Brasil e pelos EUA é possível observar diferenças entre os estilos de pesquisa desenvolvidos. Enquanto as pesquisas brasileiras parecem seguir o padrão dos demais países com menor quantidade de trabalhos, as pesquisas dos EUA apresentam maior diversidade com relação aos aspectos observados.

Em relação ao público alvo, desde 2009 os EUA promovem pesquisas com diversos públicos, envolvendo desde crianças até alunos de pós-graduação, tanto de cur-

\footnotetext{
${ }^{12}$ Computação Desplugada: 6, 9, 10, 14 , 15, 16, 17, 22, 30, 32, 38, 39, 41, 42, 44 e 45.

${ }^{13}$ Disponível em: https://scratch.mit.edu/.

${ }^{14}$ Scratch: 3, 5, 12, 16, 19, 20, 22, 25, 28, 30, 45 e 46.

${ }^{15}$ Kit de Robótica: 10, 12, 13, 23, 24, 29, 34, e 44.

${ }^{16} 1$ semestre: $1,2,3,4,6,7,8,13,17,19,40$ e 42 .

${ }^{17} 4-7$ encontros: 10, 12, 15, 20, 21, 26, 27, 29, 30, 32 e 43.
} 
VII Congresso Brasileiro de Informática na Educação (CBIE 2018)

Anais do XXIX Simpósio Brasileiro de Informática na Educação (SBIE 2018)

sos relacionados à Ciência da Computação, quanto de cursos de outras áreas de estudo. O Brasil apresenta foco maior em pesquisas com estudantes da educação básica, tendo apenas uma pesquisa com público diferente (Educação de Jovens e Adultos). Portanto, explorar outros públicos é uma oportunidade de pesquisa para o Brasil. Outra diferença relevante é em relação ao tempo de duração das iniciativas: os EUA apresentam pesquisas mais longas, com duração entre 8 semanas à 1 semestre, enquanto no Brasil as pesquisas costumam ser mais pontuais, durando apenas entre 1 e 7 encontros.

Sobre os conteúdos utilizados nas atividades, as pesquisas brasileiras focam em matérias já relacionadas à computação ou em matérias muito próximas, como a matemática. Nos EUA, além de cobrir os temas diretamente relacionados à computação, as pesquisas exploram explicitamente o potencial transdisciplinar do PC, com iniciativas situadas em biologia e música, por exemplo. Desde Papert, argumenta-se que PC pode ser utilizado na resolução de problemas de diversas áreas, e desde Wing que se tem reforçado que esta habilidade é fundamental para todos, não só para cientistas da computação. Assim, explorar a transdisciplinaridade do PC realizando pesquisas com atividades contextualizadas em disciplinas não ligadas diretamente à computação parece também ser um campo a ser explorado no Brasil e nos demais países.

O contexto socioeconômico e político certamente influencia os rumos das pesquisas e pode explicar parte das diferenças identificadas entre EUA e Brasil. No que diz respeito às ferramentas utilizadas, Computação Desplugada é a mais citada, possivelmente pelo fato de não requerer um computador. Nestes casos, os pesquisadores podem utilizar de muita criatividade para envolver o público em dinâmicas sem se preocupar com a infraestrutura técnica do local, e com a possível resistência dos alunos em utilizar o computador. Em países como o Brasil, o potencial desta ferramenta se mostra ainda mais singular especialmente para iniciativas realizadas com públicos com dificuldades de acesso à escola ou em escola com poucos recursos.

Entretanto, embora reconhecido o contexto desafiador do Brasil, somente uma das pesquisas brasileiras menciona a participação de alunos em contexto desafiador (i.e., alunos da Educação de Jovens e Adultos). É possível que pesquisas em contextos desafiadores ainda estejam em estágios iniciais de desenvolvimento ou que seus autores não estejam destacando os aspectos desafiadores do contexto pesquisado. Sendo o Brasil um país com grande diversidade de população, e sendo um grande desafio da pesquisa em computação vencer barreiras tecnológicas, educacionais, culturais, sociais e econômicas para viabilizar o acesso participativo e universal do cidadão brasileiro ao conhecimento (vide Desafio \#4) [SBC 2006], parece haver necessidade de intensificar esforços de pesquisa e atuação pela comunidade brasileira de Computação no contexto desse desafio ${ }^{18}$.

$\mathrm{O}$ PC tem grande potencial de contribuir com a inclusão efetiva das pessoas. Iniciativas para promover as habilidades compreendidas pelo PC voltadas à comunidade, concebidas com as pessoas de modo a reconhecer e considerar a sua realidade e contexto, primando pelo acesso universal e participativo, podem contribuir com o avanço no desa-

\footnotetext{
${ }^{18} \mathrm{O}$ escopo dos Grandes Desafios de Pesquisa em Computação no Brasil propostos pela SBC teve abrangência de 2006-2016. Reconhecendo a relevância do Desafio \#4, Acesso Participativo e Universal do Cidadão Brasileiro ao Conhecimento, a comunidade brasileira de IHC estendeu o alcance desse desafio para 2012-2022 com o GranDIHC-BR. Relatório Técnico disponível em: http://comissoes.sbc.org.br/ceihc/wp-content/uploads/2017/10/rt_grandes_desafios_ihc_2012.pdf?x70287
} 
VII Congresso Brasileiro de Informática na Educação (CBIE 2018)

Anais do XXIX Simpósio Brasileiro de Informática na Educação (SBIE 2018)

fio \#4, pois podem tornar o cidadão um usuário que participa efetivamente da geração do conhecimento.

\section{Conclusão}

Nos últimos anos o PC se tornou um tema bastante conhecido, com diversas iniciativas de pesquisa sendo realizadas visando o desenvolvimento desta habilidade. Este artigo mapeou iniciativas realizadas dentro e fora do Brasil buscando identificar semelhanças, tendências e oportunidades de pesquisa. Para isso, um mapeamento sistemático da literatura foi realizado investigando características como: público-alvo das iniciativas, países que mais publicaram resultados, duração das iniciativas e conteúdos trabalhados, entre outros. Partindo de um conjunto de 468 artigos, 46 artigos foram selecionados após aplicação de critérios de exclusão e de qualidade.

Os artigos selecionados mostram que as propostas de iniciativas práticas para promover o PC ainda são recentes, datando de 2009, e que os EUA se destacam na quantidade e na variedade das iniciativas, abrangendo diferentes públicos, utilizando diferentes ferramentas e explorando PC em outras disciplinas e áreas. No Brasil, embora os primeiros resultados mapeados datem de 2015, indicando um estágio inicial de pesquisas no tema, já é possível observar uma expressiva quantidade de publicações, demonstrando que o país vem avançando na área. Entretanto, embora o contexto socioeconômico seja um desafio maior no cenário brasileiro, ainda não foi possível identificar se a comunidade brasileira de Computação tem direcionado esforços para pesquisas em contextos desafiadores. Como oportunidade de trabalho no Brasil, sugerimos a ampliação do público-alvo, bem como a exploração de iniciativas transdisciplinares.

\section{Agradecimentos}

O presente trabalho foi realizado com apoio da Coordenação de Aperfeiçoamento de Pessoal de Nível Superior - Brasil (CAPES) - Código de Financiamento 001.

\section{Referências}

Avila, C., Cavalheiro, S., Bordini, A., Marques, M., Cardoso, M., and Feijo, G. (2017). Metodologias de Avaliação do Pensamento Computacional: uma revisão sistemática. In Anais do XXVIII Simpósio Brasileiro de Informática na Educação (SBIE 2017).

Barr, V. and Stephenson, C. (2011). Computational thinking to k-12 : What is involved and what is the role of the computer science education community? ACM Inroads, 2(1):48-54.

Bordini, A., Avila, C., Marques, M., Foss, L., and Cavalheiro, S. (2017). Pensamento Computacional nos Ensinos Fundamental e Médio: uma revisão sistemática. In Anais do XXVIII Simpósio Brasileiro de Informática na Educação (SBIE 2017).

Carvalho, J., Netto, J. F., and Almeida, T. (2017). Revisão Sistemática de Literatura sobre Pensamento Computacional por Meio de Objetos de Aprendizagem. In Anais do XXVIII Simpósio Brasileiro de Informática na Educação (SBIE 2017).

Liz, A., Oliveira De Araújo, S., Andrade, W. L., and Serey Guerrero, D. D. (2016). Um Mapeamento Sistemático sobre a Avaliação do Pensamento Computacional no Brasil. In Anais dos Workshops do V Congresso Brasileiro de Informática na Educação (CBIE 2016). 
VII Congresso Brasileiro de Informática na Educação (CBIE 2018)

Anais do XXIX Simpósio Brasileiro de Informática na Educação (SBIE 2018)

Papert, S. (1980). Mindstorms: Children, Computers, and Powerful Ideas. Basic Books.

Petersen, K., Vakkalanka, S., and Kuzniarz, L. (2015). Guidelines for conducting systematic mapping studies in software engineering: An update. Information and Software Technology, 64:1-18.

SBC (2006). Grandes Desafios da Pesquisa em Computação no Brasil-2006-2016: relatório sobre o seminário realizado em 8 e 9 de maio de 2006. Technical report.

Wing, J. M. (2006). Computational thinking. Communications of the ACM, 49(3):33-35.

Zanetti, H., Borges, M., and Ricarte, I. (2016). Pensamento Computacional no Ensino de Programação: Uma Revisão Sistemática da Literatura Brasileira. In Anais do XXVII Simpósio Brasileiro de Informática na Educação (SBIE 2016).

\section{Apêndice A - Relação de artigos selecionados.}

Tabela 2. Relação de artigos selecionados (1/2).

\begin{tabular}{|c|c|}
\hline ID & \\
\hline 1 & $\begin{array}{l}\text { Hambrusch, S., Hoffmann, C., Korb, J. T., Haugan, M., and Hosking, A. L. (2009). A Multidisciplinary Approach Towards } \\
\text { Computational Thinking for Science Majors. Proceedings of the 40th ACM Technical Symposium on Computer Science } \\
\text { Education. }\end{array}$ \\
\hline 2 & $\begin{array}{l}\text { Qin, H. (2009). Teaching Computational Thinking through Bioinformatics to Biology Students. Proceedings of the 40th } \\
\text { ACM Technical Symposium on Computer Science Education. }\end{array}$ \\
\hline $\mathbf{3}$ & $\begin{array}{l}\text { Ruthmann, A., Heines, J. M., Greher, G. R., Laidler, P., and Saulters, II, C. (2010). Teaching Computational Thinking through } \\
\text { Musical Live Coding in Scratch. Proceedings of the 41st ACM Technical Symposium on Computer Science Education. }\end{array}$ \\
\hline 4 & $\begin{array}{l}\text { Yang, H. I., Martin, P., Satterfield, D., Babbitt, R., Wong, J., Shelley, M., and Chang,C. K. (2011). A Novel Interdisciplinary } \\
\text { Course in Gerontechnology for Disseminating Computational Thinking. Frontiers in Education Conference. }\end{array}$ \\
\hline 5 & $\begin{array}{l}\text { Wolz, U., Stone, M., Pearson, K., Pulimood, S. M., and Switzer, M. (2011). Computational Thinking and Expository Writing } \\
\text { in the Middle School. ACM Transactions on Computing Education. }\end{array}$ \\
\hline 6 & $\begin{array}{l}\text { A. Qualls, J., Grant, M., and Sherrell, L. (2011). CS1 Students' Understanding of Computational Thinking Concepts. Journal } \\
\text { of Computing Sciences in Colleges. }\end{array}$ \\
\hline 7 & $\begin{array}{l}\text { Kafura, D. and Tatar, D. (2011). Initial Experience with a Computational Thinking Course for Computer Science Students. } \\
\text { Proceedings of the 42nd ACM Technical Symposium on Computer Science Education }\end{array}$ \\
\hline 8 & $\begin{array}{l}\text { L'Heureux, J., Boisvert, D., Cohen, R., and Sanghera, K. (2012). IT Problem Solving: An Implementation of Computational } \\
\text { Thinking in Information Technology. Proceedings of the 13th Annual Conference on Information Technology Education. }\end{array}$ \\
\hline 9 & $\begin{array}{l}\text { Hsi, S. and Eisenberg, M. (2012). Math on a Sphere: Using Public Displays to Support Children's Creativity and Computati- } \\
\text { onal Thinking on 3D Surfaces. Proceedings of the 11th International Conference on Interaction Design and Children. }\end{array}$ \\
\hline 10 & $\begin{array}{l}\text { Touretzky, D. S., Marghitu, D., Ludi, S., Bernstein, D., and Ni, L. (2013). Accelerating K-12 Computational Thinking Using } \\
\text { Scaffolding, Staging, and Abstraction. Proceeding of the 44th ACM Technical Symposium on Computer Science Education. }\end{array}$ \\
\hline 11 & $\begin{array}{l}\text { Miller, L. D., Soh, L. K., Chiriacescu, V., Ingraham, E., Shell, D. F., Ramsay, S., and Hazley, M. P. (2013). Improving } \\
\text { Learning of Computational Thinking Using Creative Thinking Exercises in CS-1 Computer Science Courses. Frontiers in } \\
\text { Education Conference. }\end{array}$ \\
\hline 12 & $\begin{array}{l}\text { Webb, H. and Rosson, M. B. (2013). Using Scaffolded Examples to Teach Computational Thinking Concepts. Proceeding of } \\
\text { the 44th ACM Technical Symposium on Computer Science Education. }\end{array}$ \\
\hline 13 & $\begin{array}{l}\text { Van Dyne, M. and Braun, J. (2014). Effectiveness of a Computational Thinking (CS0) Course on Students Analytical Skills. } \\
\text { Proceedings of the 45th ACM Technical Symposium on Computer Science Education. }\end{array}$ \\
\hline 14 & $\begin{array}{l}\text { Towhidnejad, M., Kestler, C., Jafer, S., and Nicholas, V. (2014). Introducing Computational Thinking through Stealth Tea- } \\
\text { ching. Frontiers in Education Conference. }\end{array}$ \\
\hline 15 & $\begin{array}{l}\text { Hinterholz, L. and da Cruz, M. K. (2015). Desenvolvimento do Pensamento Computacional: Um relato de atividade junto ao } \\
\text { Ensino Médio, através do Estágio Supervisionado em Computação III. Anais do XXI Workshop de Informática na Escola. }\end{array}$ \\
\hline 16 & $\begin{array}{l}\text { Peel, A., Fulton, J., and Pontelli, E. (2015). DISSECT: An Experiment in Infusing Computational Thinking in a Sixth Grade } \\
\text { Classroom. Frontiers in Education Conference. }\end{array}$ \\
\hline 17 & $\begin{array}{l}\text { Nesiba, N., Pontelli, E., and Staley, T. (2015). DISSECT: Exploring the Relationship Between Computational Thinking and } \\
\text { English Literature in K-12 Curricula. Frontiers in Education Conference. }\end{array}$ \\
\hline 18 & $\begin{array}{l}\text { Rode, J. A., Weibert, A., Marshall, A., Aal, K., von Rekowski, T., El Mimouni, H., and Booker, J. (2015). From Computatio- } \\
\text { nal Thinking to Computational Making. Proceedings of the ACM International Joint Conference on Pervasive and Ubiquitous } \\
\text { Computing. }\end{array}$ \\
\hline
\end{tabular}


VII Congresso Brasileiro de Informática na Educação (CBIE 2018)

Anais do XXIX Simpósio Brasileiro de Informática na Educação (SBIE 2018)

Tabela 3. Relação de artigos selecionados (2/2).

\begin{tabular}{|c|c|}
\hline ID & Referência \\
\hline 19 & $\begin{array}{l}\text { Rodriguez, C., Zem-Lopes, A. M., Marques, L., and Isotani, S. (2015). Pensamento Computacional: transformando ideias } \\
\text { em jogos digitais usando o Scratch. Anais do XXI Workshop de Informática na Escola. }\end{array}$ \\
\hline 20 & $\begin{array}{l}\text { Ramos, F. and Teixeira, L. D. S. (2015). Significação da Aprendizagem Através do Pensamento Computacional no Ensino } \\
\text { Médio: uma Experiência com Scratch. Anais do XXI Workshop de Informática na Escola. }\end{array}$ \\
\hline 21 & $\begin{array}{l}\text { Fronza, I., El Ioini, N., and Corral, L. (2015). Students Want to Create Apps: Leveraging Computational Thinking to Teach } \\
\text { Mobile Software Development. Proceedings of the 16th Annual Conference on Information Technology Education. }\end{array}$ \\
\hline 22 & $\begin{array}{l}\text { Schoeffel, P., Moser, P., Varela, G., Durigon, L., de Albuquerque, G. C., and Niquelatti, M. (2015). Uma Experiência no } \\
\text { Ensino de Pensamento Computacional e Fomento à Participação na Olimpíada Brasileira de Informática com Alunos do } \\
\text { Ensino Fundamental. Anais dos Workshops do IV Congresso Brasileiro de Informática na Educação. }\end{array}$ \\
\hline 23 & $\begin{array}{l}\text { Chaudhary, V., Agrawal, V., Sureka, P., and Sureka, A. (2016). An Experience Report on Teaching Programming and } \\
\text { Computational Thinking to Elementary Level Children using Lego Robotics Education Kit. 8th International Conference on } \\
\text { Technology for Education. }\end{array}$ \\
\hline 24 & $\begin{array}{l}\text { da Silva, D. P., Sidnei, S., Jesus, A., and Silva, C. E. P. (2016). Aplicação de Robótica na Educação de Forma Gradual para o } \\
\text { Estímulo do Pensamento Computacional. Anais dos Workshops do V Congresso Brasileiro de Informática na Educação. }\end{array}$ \\
\hline 25 & $\begin{array}{l}\text { Mason, D., Khan, I., and Farafontov, V. (2016). Computational Thinking as a Liberal Study. Proceedings of the 47th ACM } \\
\text { Technical Symposium on Computing Science Education. }\end{array}$ \\
\hline 26 & $\begin{array}{l}\text { Segredo, E., Miranda, G., León, C., and Santos, A. (2016). Developing Computational Thinking Abilities Instead of Digital } \\
\text { Literacy in Primary and Secondary School Students. Smart Education and e-Learning. }\end{array}$ \\
\hline 27 & $\begin{array}{l}\text { Guo, Y., Wagh, A., Brady, C., Levy, S. T., Horn, M. S., and Wilensky, U. (2016). Frogs to Think with: Improving Students' } \\
\text { Computational Thinking and Understanding of Evolution in A Code-First Learning Environment. Proceedings of the 15th } \\
\text { International Conference on Interaction Design and Children. }\end{array}$ \\
\hline 28 & $\begin{array}{l}\text { Munoz, R., Barcelos, T. S., Villarroel, R., and Silveira, I. F. (2016). Game Design Workshop to Develop Computational } \\
\text { Thinking Skills in teenagers with Autism Spectrum Disorders. Proceedings of the 11th Iberian Conference on Information } \\
\text { System and Technologies. }\end{array}$ \\
\hline 29 & $\begin{array}{l}\text { Oliveira, E. and Araujo, A. L. (2016). Pensamento Computacional e Robótica: Um Estudo Sobre Habilidades Desenvolvidas } \\
\text { em Oficinas de Robótica Educacional. Anais do XXVII Simpósio Brasileiro de Informática na Educação. }\end{array}$ \\
\hline 30 & $\begin{array}{l}\text { Bathke, J. d. S. and Raabe, A. (2016). Pensamento Computacional na Educacão de Jovens e Adultos: Lições Aprendidas. } \\
\text { Anais dos Workshops do V Congresso Brasileiro de Informática na Educação. }\end{array}$ \\
\hline 31 & $\begin{array}{l}\text { Pinho, G., Weisshahn, Y., Reiser, R., de Brum, C. F., Cavalheiro, S., Foss, L., Aguiar, M.,and Bois, A. D. (2016). Pensamento } \\
\text { Computacional no Ensino Fundamental: Relato de Atividade de Introdução a Algoritmos. Anais do XXII Workshop de } \\
\text { Informática na Escola). }\end{array}$ \\
\hline 32 & $\begin{array}{l}\text { Pinho, G., Weisshahn, Y., de Brum, C. F., Cavalheiro, G. G. H., and Cavalheiro, S. (2016). Proposta de Jogo Digital para } \\
\text { Dispositivos Móveis: Desenvolvendo Habilidades do Pensamento Computacional. Anais do XXVII Simpósio Brasileiro de } \\
\text { Informática na Educação. }\end{array}$ \\
\hline 33 & $\begin{array}{l}\text { Li, W.-L., Hu, C.-F., and Wu, C.-C. (2016). Teaching High School Students Computational Thinking with Hands-on Activi- } \\
\text { ties. Proceedings of the ACM Conference on Innovation and Technology in Computer Science Education. }\end{array}$ \\
\hline 34 & $\begin{array}{l}\text { Leonard, J., Buss, A., Gamboa, R., Mitchell, M., Fashola, O. S., Hubert, T., and Al-mughyirah, S. (2016). Using Robotics and } \\
\text { Game Design to Enhance Children's Self-Efficacy, STEM Attitudes, and Computational Thinking Skills. Journal of Science } \\
\text { Education and Technology. }\end{array}$ \\
\hline 35 & $\begin{array}{l}\text { Gautam, A., Bortz, W. E. W., and Tatar, D. (2017). Case for Integrating Computational Thinking and Science in a Low- } \\
\text { Resource Setting. Proceedings of the 9th International Conference on Information and Communication Technologies and } \\
\text { Development. }\end{array}$ \\
\hline 36 & $\begin{array}{l}\text { Krugel, J. and Hubwieser, P. (2017). Computational Thinking as Springboard for Learning Object-Oriented Programming in } \\
\text { an Interactive MOOC. Global Engineering Education Conference. }\end{array}$ \\
\hline 37 & $\begin{array}{l}\text { Corradini, I., Lodi, M., and Nardelli, E. (2017). Computational Thinking in Italian Schools: Quantitative Data and Teachers' } \\
\text { Sentiment Analysis after Two Years of "Programma il Futuro"Project. Proceedings of the ACM Conference on Innovation } \\
\text { and Technologyin Computer Science Education. }\end{array}$ \\
\hline 38 & $\begin{array}{l}\text { Brackmann, C. P., Román-González, M., Robles, G., Moreno-León, J., Casali, A., and Barone, D. (2017). Development } \\
\text { of Computational Thinking Skills through Unplugged Activities in Primary School. Proceedings of the 12th Workshop on } \\
\text { Primary and Secondary Computing Education. }\end{array}$ \\
\hline 39 & $\begin{array}{l}\text { Cândido, D., Pessoa, G., Vasconcelos, B., da Silva, L. L., Oliveira, R., Oliveira, M., and Falcão, T. P. (2017). Estudo } \\
\text { Comparativo de Abordagens Referentes ao Desenvolvimento do Pensamento Computacional. Anais do XXIII Workshop de } \\
\text { Informática na Escola. }\end{array}$ \\
\hline 40 & $\begin{array}{l}\text { Senske, N. (2017). Evaluation and Impact of a Required Computational Thinking Course for Architecture Students. Procee- } \\
\text { dings of the ACM Technical Symposium on Computer Science Education. }\end{array}$ \\
\hline 41 & $\begin{array}{l}\text { Rodrigues, G. and Sousa, L. (2017). O ensino do Pensamento Computacional como forma de inclusão tecnológica e } \\
\text { motivação de crianças. Anais do XXVIII Simpósio Brasileiro de Informática na Educação. }\end{array}$ \\
\hline 42 & $\begin{array}{l}\text { Reis, F. D. M., Cristiano, F., Martins, D., and Rocha, P. D. (2017). Pensamento Computacional: Uma Proposta de Ensino } \\
\text { com Estratégias Diversificadas para Crianças do Ensino Fundamental. Anais do XXIII Workshop de Informática na Escola. }\end{array}$ \\
\hline 43 & $\begin{array}{l}\text { Chiazzese, G., Fulantelli, G., Pipitone, V., and Taibi, D. (2017). Promoting computational thinking and creativeness in } \\
\text { primary school children. Proceedings of the 5th International Conference on Technological Ecosystems for Enhancing Mul- } \\
\text { ticulturality. }\end{array}$ \\
\hline 44 & $\begin{array}{l}\text { Conde, M. A., Fernández-Llamas, C., Rodríguez-Sedano, F. J., Guerrero-Higueras, A. M., Matellán-Olivera, V., and García- } \\
\text { Peñalvo, F. J. (2017). Promoting Computational Thinking in K-12 students by applying unplugged methods and robotics. } \\
\text { Proceedings of the 5th International Conference on Technological Ecosystems for Enhancing Multiculturality. }\end{array}$ \\
\hline 45 & $\begin{array}{l}\text { Fronza, I., Ioini, N. E., and Corral, L. (2017). Teaching Computational Thinking Using Agile Software Engineering Methods: } \\
\text { A Framework for Middle Schools. ACM Transactions on Computing Education. }\end{array}$ \\
\hline 41 & $\begin{array}{l}\text { Santana, A. L. M., de Jesus, E. A., Raabe, A., Santana, L., Cucco, L., and Ramos, G. (2017). Tem Ideia na Rede: Inserindo o } \\
\text { Pensamento Computacional na Rede Municipal de Ensino. Anais do XXIII Workshop de Informática na Escola. }\end{array}$ \\
\hline
\end{tabular}

